\title{
Therapeutic Mammotome excision of fibroadenomas
}

\author{
A. J. Maxwell \\ Dr Anthony J Maxwell, Consultant Radiologist, Breast Unit, Royal Bolton Hospital, Minerva Road, \\ Farnworth, Bolton BL4 OJR, UK.
}

\begin{abstract}
Fibroadenomas are most common benign tumour of the breast, usually affecting pre-menopausal women. The traditional treatment aimed at symptomatic relief and confirmation of the lesion's benign nature through open surgical excision and biopsy. The rise of 'triple assessment' in recent years has meant that a definitive benign diagnosis is now generally possible without recourse to open biopsy. But patients would still face undergoing open surgical excision for relief of symptoms. A third option is now available - that of percutaneous vacuum-assisted excision under local anaesthetic. Performed in a similar fashion to conventional percutaneous needle biopsy, the procedure can normally be completed within $15 \mathrm{~min}$ and offers quicker recovery times and better cosmetic outcome than traditional excision.
\end{abstract}

Keywords: Benign breast tumours; Fibroadenomas; Mammotome; Percutaneous vacuum-assisted excision

\section{Introduction}

Fibroadenomas are the commonest benign tumour of the breast, usually presenting as solitary lumps in young women. They seldom present clinically after the menopause, although they are often discovered during routine mammographic screening and may require biopsy to exclude malignancy.

The traditional management of symptomatic fibroadenomas has been by open surgical excision biopsy, allowing both relief from symptoms (although at the expense of a surgical scar) and histological confirmation of their benign nature. With the advent of 'triple assessment' in recent years, a definitive benign diagnosis is now generally possible without recourse to open biopsy. This then leaves the surgeon and

Correspondence to: Dr AJ Maxwell, Consultant Radiologist, Royal Bolton Hospital. Tel: +01204 390387/010/397. E-mail: anothony.maxwell@rbh. nhs.uk

Received: 02/10/05

Revised: 09/11/05

Accepted: 10/11/05

First published online 17/02/06

BCO/485/2005/FO patient with a choice - either to leave the lump alone, knowing that it is benign, or to proceed with surgical excision for relief of symptoms. A third option is now available - that of percutaneous vacuum-assisted excision under local anaesthetic.

\section{The Mammotome device}

Several manufacturers produce vacuum-assisted biopsy devices, of which the first and most widely used is the Mammotome from Ethicon Endo-Surgery (Cincinnati, Ohio, USA). The Mammotome was originally developed as a large bore percutaneous biopsy device primarily for use under stereotactic $X$-ray guidance, but technical improvements allow the latest devices to be used for therapeutic excision of benign lesions in addition to their diagnostic role.

The Mammotome biopsy needle (known as the probe) consists of a large bore needle of ovoid cross section containing two channels. The larger of these is the specimen channel and is circular in section with a side aperture near the tip. The smaller channel is the vacuum channel, and this opens via a series of 
small holes into the base of the specimen aperture. Running within the specimen channel is a rotating cutting cannula which advances to the tip of the outer cannula during tissue removal. The probe is integrated into a plastic base unit, the whole assembly being for one time use. The probe assembly fits into a mounting device known as a holster which is connected by one or more cables and two vacuum tubes to the base unit. This consists of a floor-standing unit containing a vacuum pump, disposable plastic vacuum canister, electric motor and computerised control unit.

Holsters are available in both stereotactic and handheld (ultrasound guided) versions, and 8-, 11and 14-gauge probes are available. Therapeutic fibroadenoma excision is generally carried out under ultrasound guidance using the handheld holster and probe. The new Mammotome EX system is a considerable improvement on the earlier version of the handheld holster and probe, as it has an integral drive motor, allowing elimination of the heavy mechanical drive cables. The author's personal preference is to perform ultrasound-guided excisions using an 8gauge probe for all but the smallest lesions. This allows removal of a relatively large volume of tissue with each advance of the cutting cannula, reducing the number of passes required, minimising the procedure time and increasing the likelihood of complete lesion excision.

The Mammotome operates in three modes positioning, sampling and probe clearance - and can be controlled either by buttons on the handheld holster or by foot pedals. In positioning mode the cutting cannula can be advanced to close the specimen aperture without removing tissue, allowing the probe to be inserted into or withdrawn from the breast. In sampling mode, pressing the 'forward' button or foot pedal will result in the application of a vacuum to the base of the specimen aperture, drawing tissue into the aperture which can then be severed by the advancing rotating cutting cannula. Depression of the 'back' button or pedal will result in the withdrawal of the cutting cannula and the delivery of the specimen into the specimen collection chamber. Probe blockages can be cleared with vacuum pulses in the 'probe clear' mode.

\section{Performing the procedure}

Percutaneous therapeutic Mammotome excision of fibroadenomas is performed in a similar fashion to conventional percutaneous needle biopsy. A suitable site for skin puncture some distance away from the lesion is chosen according to the position of the lesion in the breast and the Mammotome and ultrasound probe orientations preferred by the operator. It is preferable to plan the Mammotome track to be as nearly parallel to the chest wall as possible, but without making the track excessively long. Local anaesthetic is infiltrated into the skin and subcutaneous tissues, and a small skin incision no larger than the tip of the probe is made in the skin. Further local anaesthetic is then injected into the deeper tissue using a spinal or similar needle positioned under direct ultrasonic visualisation, infiltrating along the planned Mammotome track and around the lesion. Ten millilitres of $1 \%$ or $2 \%$ Xylocaine with 1 in 200000 adrenaline is usually adequate. For a lesion in a small breast and/or a lesion close to the chest wall it is often helpful to lift the lesion away from the chest wall by injecting a 'pad' of anaesthetic posterior to it.

The probe is inserted in 'positioning' mode, with the aperture closed. A blade on the tip of 8-gauge probe (and available as an option on 11-gauge probes) helps it to pass cleanly through the tissues. The probe is generally positioned so that the aperture (which can be readily identified on ultrasound, even in the closed position) lies immediately behind the lesion, although some larger lesions may be more easily removed by being transfixed by the probe. The system is then switched to 'sampling' mode, and lesion removal can begin. If the probe is correctly positioned posterior to the lesion, the mass can be seen being sucked into the specimen aperture as the 'forward' button or pedal is depressed.

Progress of lesion removal is usually well seen on ultrasound, although occasionally completeness of removal can be difficult to assess due to the presence of air and/or blood in the cavity. It is often necessary to take cores through an arc of up to $180^{\circ}$ (or $360^{\circ}$ for transfixed lesions) in order to maximise the likelihood of complete lesion removal. This is done by incrementally rotating the holster between samplings when the probe aperture is closed. In addition, elevation of the probe as the lesion is progressively removed may help the remaining part of the lesion to enter the aperture. It is occasionally necessary to withdraw and reposition the probe to obtain complete excision of larger lesions.

The number of cores required to obtain complete lesion removal is very variable, depending on the size of the lesion, the gauge of probe used and the position of the probe relative to the lesion. Most cases will require between 5 and 20 cores to be taken. This can be done very quickly with the new Mammotome EX system, the rate limiting step often being the speed with which the assistant can remove the cores from the specimen collection chamber.

Procedure times of $10-60 \mathrm{~min}$ are quoted in the literature [1,2] but in the author's experience (using an 8-gauge probe where indicated) most therapeutic excisions can be completed within $15 \mathrm{~min}$. The procedure is tolerated remarkably well by the majority 
of patients $[3,4]$, in part due to elimination of the need for repeated insertions of the device compared to conventional 14-gauge core biopsy.

Following withdrawal of the probe, firm pressure is applied for several minutes to the area of tissue removal (not the skin incision, which is some distance away). A pressure dressing is then applied. We advise the patient to rest at home for the remainder of the day, and to remove the dressing the following morning.

\section{How good is mammotomy at removing fibroadenomas?}

The likelihood of complete lesion removal is dependent on the size of the fibroadenoma and the gauge of the Mammotome probe used. There are relatively few published papers on the subject of ultrasoundguided Mammotome removal of breast masses, and most of these relate to the use of the 11-gauge probe. There is, however, reasonable consensus in the literature that complete excision of lesions $15 \mathrm{~mm}$ or less in diameter can be achieved in the majority of cases with the 11-gauge probe $[1,2,5,6,7]$. Lesions over $20 \mathrm{~mm}$ in diameter are unlikely to be completely removed with this probe [2], and here an 8-gauge probe is more likely to be successful [7].

The likelihood of recurrence of a fibroadenoma after Mammotome excision appears small. Sperber [2] performed follow-up ultrasound at 2 years on 43 women who had apparently completely excised fibroadenomas and found no recurrent lesions, and Huber [3] found no recurrences on ultrasound at 6 months in 41 women. March [4], however, found $38 \%$ of lesions still to be visible at 6 months, although not all of these were fibroadenomas. Mammotomy with apparently complete lesion removal at the time of the procedure seems to be effective for elimination of a palpable mass in virtually all cases.

\section{Advantages and disadvantages of the Mammotome procedure}

The main advantages of Mammotome removal of fibroadenomas is that the procedure can be performed on an outpatient basis under local anaesthetic and that it results in minimal scarring. The great majority of patients are satisfied with the procedure and its cosmetic result $[4,7]$. Even with the cost of the probe and other disposable items (around $£ 250$ ) the procedure is substantially cheaper than a day case operation under local anaesthetic, although the capital costs of the base unit and holster are significant.

In young women with the typical clinical and radiological features of a fibroadenoma, and who wish to have the lump removed, it would not be unreasonable to perform therapeutic Mammotome excision of the lesion as the primary definitive diagnostic procedure, avoiding the need for core biopsy or fine needle aspiration cytology.

Significant complications of mammotomy are uncommon. Bleeding during the procedure is seldom a problem as blood is automatically aspirated from the cavity during tissue sampling. Addition suction can be applied between samplings if necessary. Occasionally, however, bleeding may obscure the lesion and require termination of the procedure. Bruising following mammotomy is usually more marked than following 14-gauge core biopsy, but persisting haematomas which either require evacuation or persist symptomatically occur in fewer than $2 \%$ of cases [1,5]. Vasovagal reactions occur occasionally. Infection following the procedure is rare.

\section{Conclusion}

Mammotome excision of fibroadenomas offers a viable safe alternative to traditional surgical excision, with reduced costs and an excellent cosmetic result. It offers greater convenience for the patient, being performed as an outpatient rather than day case procedure. It does, however, require a significant capital investment in equipment, together with the availability of suitably trained personnel.

An overview of image-guided vacuum-assisted excision biopsy of benign breast lesions has recently been prepared [8] for consideration by the Interventional Procedures Advisory Committee of the UK National Institute for Clinical Excellence (NICE), which is due to report in November 2005.

\section{References}

1. Perez-Fuentes JA, Longobardi IR, Acosta VF, et al. Sonographically guided directional vacuum-assisted breast biopsy: preliminary experience in Venezuela. AJR Am J Roentgenol 2001; 177: 1459-1463.

2. Sperber F, Blank A, Metser U, et al. Diagnosis and treatment of breast fibroadenomas by ultrasound-guided vacuum-assisted biopsy. Arch Surg 2003; 138: 796-800.

3. Huber S, Wagner M, Medl M, Czembirek H. Benign breast lesions: minimally invasive vacuum-assisted biopsy with 11-gauge needles - patient acceptance and effect on follow-up imaging findings. Radiology 2003; 226: 783-790.

4. March DE, Coughlin BF, Barham RB, et al. Breast masses: removal of all US evidence during biopsy by using a handheld vacuum-assisted device - initial experience. Radiology 2003; 227: 549-555.

5. Parker SH, Klaus AJ, McWey PJ, et al. Sonographically guided directional vacuum-assisted breast biopsy using a handheld device. AJR Am J Roentgenol 2001; 177: 405-408.

6. Baez E, Huber A, Vetter M, Hackeloer BJ. Minimal invasive complete excision of benign breast tumors using a 
three-dimensional ultrasound-guided mammotome vacuum device. Ultrasound Obstet Gynecol 2003; 21: 267-272.

7. Fine RE, Boyd BA, Whitworth PW, et al. Percutaneous removal of benign breast masses using a vacuumassisted hand-held device with ultrasound guidance. Am J Surg 2002; 184: 332-336.
8. National Institute for Clinical Excellence Interventional Procedures Programme. Interventional procedures overview of image-guided vacuum assisted excision biopsy of benign breast lesions. http://www.nice.org.uk/ pdf/ip/188\%20-\%20overview\%20for\%20web.pdf 